\title{
3D PROCEDURE FOR LAYER VELOCTY ANALYSIS - COUPLED MAP MIGRATION/COHERENCY MEASURING
}

VALERY SORIN and EVGENY LANDA

IPRG, PO Box 2286, Holon 58122, Israel

Overview

A technique of layer velocity estimation through semblance analysis was reported in Hadley et al. (1988) and Landa et al. (1989). Its basic premise is that a coherency measure on unstacked seismograms reaches a maximum along reflection time trajectories that correspond to the correctly estimated velocity-depth model.

In this presentation we consider a.3-D implementation of the technique. This is an outline of the processing procedure. Input consists of interpreted zero-offset time maps and unstacked trace gathers (CMP or CS) of an arbitrary acquisition geometry. Velocity analysis is conducted for one layer at a time at a number of selected locations. In a current target layer at a certain location, the velocity is analyzed as single-parameter testing within a velocity range. For each trial velocity value, a reflector position is constructed by time-to-depth map migration using the zero-offset time map. Next, nonzero-offset reflection times for specified trace gathers are calculated by 3-D ray tracing accounting for a layered overburden. The semblance coherency measure is computed by stacking the input trace gathers along the calculated reflection traveltimes. Finally, velocity estimates in a layer are chosen corresponding to maximum semblance values.

\section{Implementation}

Regarding the described procedure of layer velocity analysis we distinguish the two following steps. For each trial velocity in a current target layer: 1) a 3-D reflector depth position is constructed from a zero-offset time map; 2) nonzero-offset reflection times are calculated and 
coherency is measured on unstacked gathers. A repeated sequence of coupled reflector construction/coherency measuring composes the main computational kernel of the procedure.

The efficiency and stability of the procedure are affected by the used techniques of map migration and travel time calculation in a multi-layered model. For these two processes we implemented a ray tracing algorithm (Sattlegger, 1985; Moser, 1991) based on Fermat's principle. The algorithm substantially exploits the network structure of a model. Interfaces in the model are represented by depth values in nodes of a 2-D grid. The velocity in each layer is specified in nodes of a 3-D grid. Rays are allowed to travel within the model between the nodes only. Ray paths are chosen according to Fermat's principle as the least-time paths.

In contrast to Snell-based techniques that are strongly sensitive to the accuracy of the derivatives of timefield and interfaces, the implemented algorithm of ray tracing does not uses the derivatives. The computational efficiency of the algorithm is achieved through its ability to restart a downward continuation of the timefield from an intermediate interface.

\section{Application}

The application of the presented procedure of layer velocity analysis is demonstrated in an offshore area of Israel. Three principal horizons were picked on stacked time sections of line survey profiles. A bathymetric map, the three constructed time maps and CMP trace gathers served an input to the procedure.

Results of 3-D velocity analysis in the third layer along one of the line profiles are presented in Fig. 1 in the form of a semblance plot. Velocity analysis was controlled by the independent estimation at the profiles intersection. From the velocity estimates obtained at the profiles, velocity maps were subsequently constructed for the three considered layers. A part of the depth model reconstructed using the velocity maps is shown in Fig. 2. 


\section{Discussion and conclusions}

The procedure was developed for layer velocity estimation in a 3-D model.

The estimates correctness is controlled by the fit of the model to the input data, namely the correspondence to the zero-offset time maps and maximization of the coherency measure on the unstacked gathers.

The procedure requires the preliminary construction of zero-offset time maps (migrated or unmigrated). In practice, the zero-offset time values are obtained from picking reflections in the stacked domain. Notice that this time information is usually the most reliable when compared with other parameters such as nonzero-offset times, ray parameters, NMO velocities, etc., frequently used in kinematic inversions.

In the developed procedure, the repeating sequence of coupled map migration/coherency measuring is used for testing of a layer velocity. The construction of the reflector depth position by map migration, separate from coherency measuring, is available due to the use of zero-offset time maps. The input gathers are used only in coherency measuring; they can be of an arbitrary acquisition geometry and, in particular, be positioned at line profiles. The implemented ray tracing algorithm based on Fermat's principle provides the robustness and efficiency of the procedure.

\section{References}

Hadley, D.M., Thorson, J. and Maher, S. 1988. Increasing interpretation accuracy: A new approach to interval velocity estimation. Leading Edge 7(9), 13-16.

Landa, E., Beydoun, W. and Tarantola, A. 1989. Reference velocity model estimation from prestack wave forms: Coherency optimization by simulated annealing. Geophysics 54, 984-990.

Moser, T.J. 1991. Shortest path calculation of seismic rays. Geophysics 56, 59-67.

Sattlegger, J.W. 1985. Map migration and modeling algorithm. 55th SEG Meeting, Washington D.C., Expanded Abstracts, 553-554. 
CMP

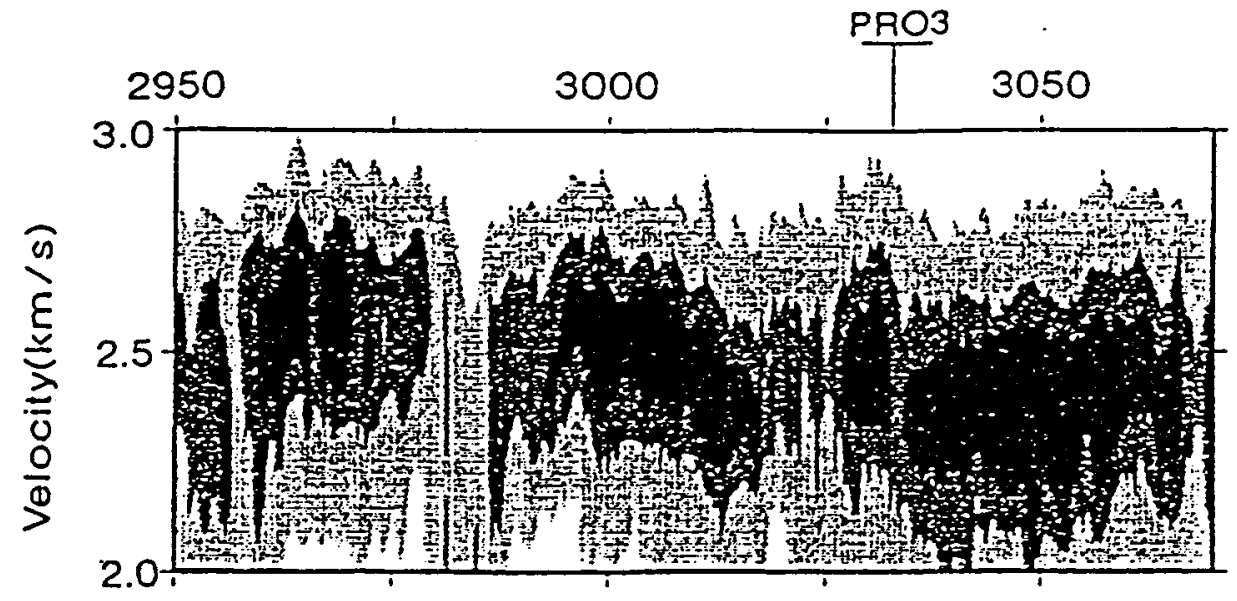

Semblance

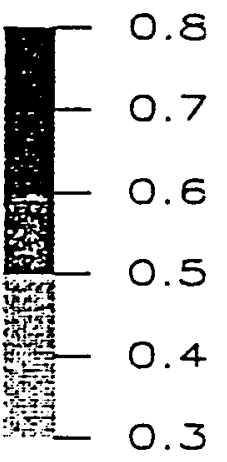

Fig.1 Semblance plot of 3-D layer velocity analysis

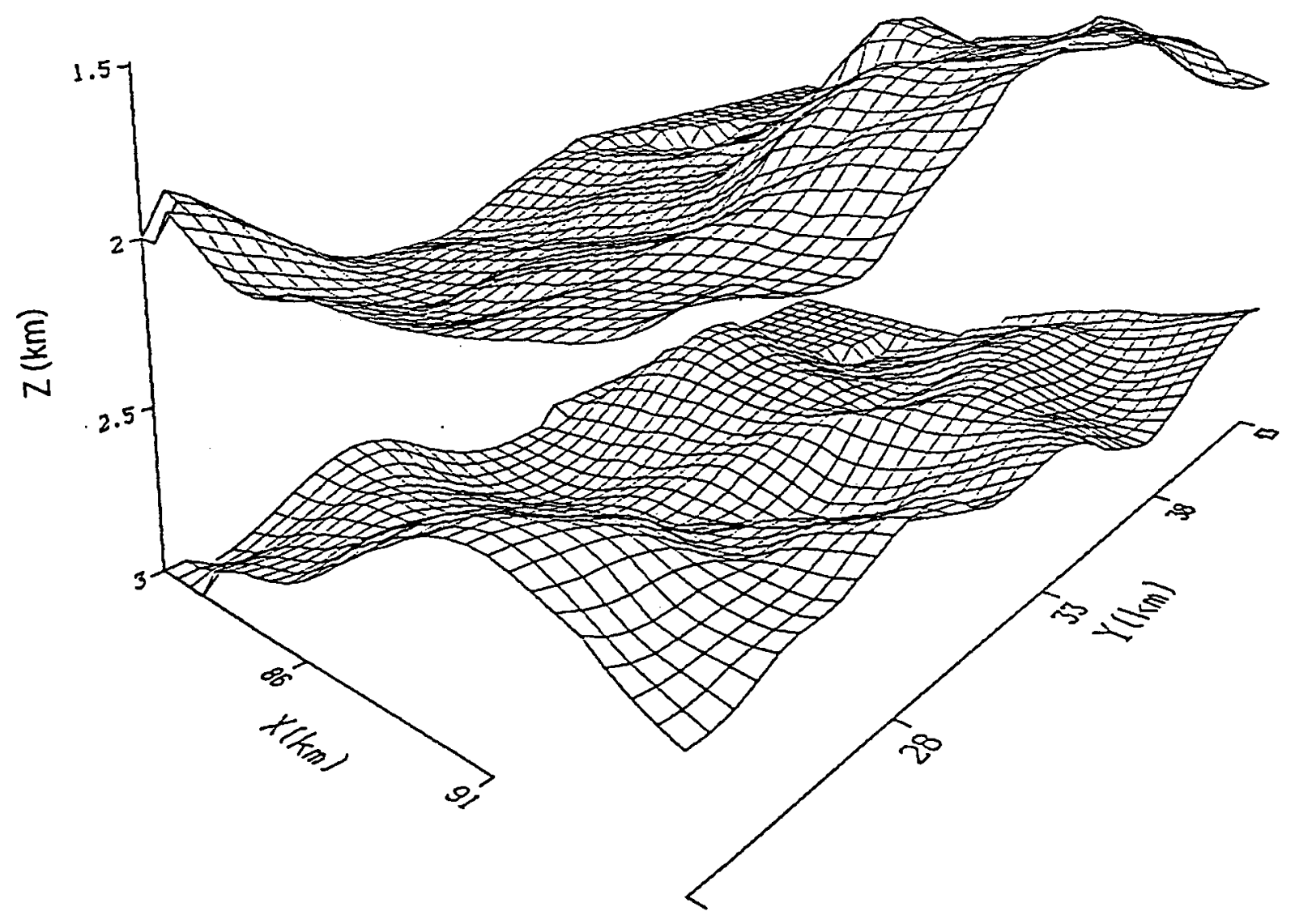

Fig.2 3-D depth model 